\title{
Convergence of the modified Mann's iterative method for asymptotically $\kappa$-strictly pseudocontractive mappings
}

Ying Zhang ${ }^{1,2^{*}}$ and Zhiwei $\mathrm{Xie}^{3}$

\author{
* Correspondence: \\ spzhangying@126.com \\ ${ }^{1}$ School of Mathematics and \\ Physics, North China Electric Power \\ University, Baoding, Hebei 071003, \\ P.R. China \\ Full list of author information is \\ available at the end of the article
}

\begin{abstract}
Let $E$ be a real uniformly convex Banach space which has the Fréchet differentiable norm, and $K$ a nonempty, closed, and convex subset of $E$. Let $T: K \rightarrow K$ be an asymptotically $\kappa$-strictly pseudocontractive mapping with a nonempty fixed point set. We prove that $(I-T)$ is demiclosed at 0 and obtain a weak convergence theorem of the modified Mann's algorithm for T under suitable control conditions. Moreover, we also elicit a necessary and sufficient condition that guarantees strong convergence of the modified Mann's iterative sequence to a fixed point of $T$ in a real Banach spaces with the Fréchet differentiable norm.

2000 AMS Subject Classification: 47H09; 47H10.
\end{abstract}

Keywords: asymptotically $k$-strictly pseudocontractive mappings, demiclosedness principle, the modified Mann's algorithm, fixed points

\section{Introduction}

Let $E$ and $E^{*}$ be a real Banach space and the dual space of $E$, respectively. Let $K$ be a nonempty subset of $E$. Let $J$ denote the normalized duality mapping from $E$ into $2^{E_{*}}$ given by $J(x)=\left\{f \in E^{*}:\langle x, f\rangle=\|x\|^{2}=\|f\|^{2}\right\}$, for all $x \in E$, where $\langle, \cdot$,$\rangle denotes the dua-$ lity pairing between $E$ and $E^{*}$. In the sequel, we will denote the set of fixed points of a mapping $T: K \rightarrow K$ by $F(T)=\{x \in K: T x=x\}$.

A mapping $T: K \rightarrow K$ is called asymptotically $\kappa$-strictly pseudocontractive with sequence $\left\{\kappa_{n}\right\}_{n=1}^{\infty} \subseteq[1, \infty)$ such that $\lim _{n \rightarrow \infty} \kappa_{n}=1$ (see, e.g., [1-3]) if for all $x, y \in K$, there exist a constant $\kappa \in[0,1)$ and $j(x-y) \in J(x-y)$ such that

$$
\left\langle T^{n} x-T^{n} y, j(x-y)\right\rangle \leq \kappa_{n}\|x-y\|^{2}-\kappa\left\|x-y-\left(T^{n} x-T^{n} y\right)\right\|^{2}, \forall n \geq 1
$$

If $I$ denotes the identity operator, then (1) can be written as

$$
\left\langle\left(I-T^{n}\right) x-\left(I-T^{n}\right) y, j(x-\gamma)\right\rangle \geq \kappa\left\|\left(I-T^{n}\right) x-\left(I-T^{n}\right) y\right\|^{2}-\left(\kappa_{n}-1\right)\|x-\gamma\|^{2}, \forall n \geq 1 .
$$

The class of asymptotically $\kappa$-strictly pseudocontractive mappings was first introduced in Hilbert spaces by Qihou [3]. In Hilbert spaces, $j$ is the identity and it is shown by Osilike et al. [2] that (1) (and hence (2)) is equivalent to the inequality

$$
\left\|T^{n} x-T^{n} y\right\|^{2} \leq \lambda_{n}\|x-y\|^{2}+\lambda\left\|x-y-\left(T^{n} x-T^{n} y\right)\right\|^{2},
$$

\section{Springer}

(c) 2011 Zhang and Xie; licensee Springer. This is an Open Access article distributed under the terms of the Creative Commons Attribution License (http://creativecommons.org/licenses/by/2.0), which permits unrestricted use, distribution, and reproduction in any medium, provided the original work is properly cited. 
where $\lim _{n \rightarrow \infty} \lambda_{n}=\lim _{n \rightarrow \infty}\left[1+2\left(\kappa_{n}-1\right)\right]=1, \lambda=(1-2 \kappa) \in[0,1)$.

A mapping $T$ with domain $D(T)$ and range $R(T)$ in $E$ is called strictly pseudocontractive of Browder-Petryshyn type [4], if for all $x, y \in D(T)$, there exists $\kappa \in[0,1)$ and $j(x-y) \in J$ $(x-y)$ such that

$$
\langle T x-T y, j(x-y)\rangle \leq\|x-y\|^{2}-\kappa\|x-y-(T x-T y)\|^{2} .
$$

If $I$ denotes the identity operator, then (3) can be written as

$$
\langle(I-T) x-(I-T) y, j(x-\gamma)\rangle \geq \kappa\|(I-T) x-(I-T) y\|^{2} .
$$

In Hilbert spaces, (3) (and hence (4)) is equivalent to the inequality

$$
\|T x-T y\|^{2} \leq\|x-y\|^{2}+k\|x-y-(T x-T y)\|^{2}, k=(1-2 \kappa)<1,
$$

It is shown in [5] that the class of asymptotically $\kappa$-strictly pseudocontractive mappings and the class of $\kappa$-strictly pseudocontractive mappings are independent.

A mapping $T$ is said to be uniformly $L$-Lipschitzian if there exists a constant $L>0$ such that

$$
\left\|T^{n} x-T^{n} y\right\| \leq L\|x-y\|, n \geq 1
$$

for all $x, y \in K$ and is said to be demiclosed at a point $p$ if whenever $\left\{x_{n}\right\} \subset D(T)$ such that $\left\{x_{n}\right\}$ converges weakly to $x \in D(T)$ and $\left\{T x_{n}\right\}$ converges strongly to $p$, then $T x=p$.

Kim and $\mathrm{Xu}$ [6] studied weak and strong convergence theorems for the class of asymptotically $\kappa$-strictly pseudocontractive mappings in Hilbert space. They obtained a weak convergence theorem of modified Mann iterative processes for this class of mappings. Moreover, a strong convergence theorem was also established in a real Hilbert space by hybrid projection method. They proved the following.

Theorem KX [6] Let $K$ be a closed and convex subset of a Hilbert space $H$. Let $T$ : $K \rightarrow K$ be an asymptotically $\kappa$-strictly pseudocontractive mapping for some $0 \leq \kappa<1$ with sequence $\left\{\kappa_{n}\right\} \subset[1, \infty)$ such that $\sum_{n=1}^{\infty}\left(\kappa_{n}-1\right)<\infty$ and $F(T) \neq \varnothing$. Let $\left\{x_{n}\right\}_{n=1}^{\infty}$ be a sequence generated by the modified Mann's iteration method:

$$
x_{n+1}=\alpha_{n} x_{n}+\left(1-\alpha_{n}\right) T^{n} x_{n}, n \geq 1,
$$

Assume that the control sequence $\left\{\alpha_{n}\right\}_{n=1}^{\infty}$ is chosen in such a way that $\kappa+\lambda \leq \alpha_{n} \leq$ $1-\lambda$ for all $n$, where $\lambda \in(0,1)$ is a small enough constant. Then, $\left\{x_{n}\right\}$ converges weakly to a fixed point of $T$.

The modified Mann's iteration scheme was introduced by Schu $[7,8]$ and has been used by several authors (see, for example, [1-3,9-11]). One question is raised naturally: is the result in Theorem KX true in the framework of the much general Banach space?

Osilike et al. [5] proved the convergence theorems of modified Mann iteration method in the framework of $q$-uniformly smooth Banach spaces which are also uniformly convex. They also obtained that a modified Mann iterative process $\left\{x_{n}\right\}$ converges weakly to a fixed point of $T$ under suitable control conditions. However, the control sequence $\left\{\alpha_{n}\right\} \subset[0,1]$ depended on the Lipschizian constant $L$ and excluded the natural choice $\alpha_{n}=\frac{1}{n}, n \geq 1$. These are motivations for us to improve the results. 
We prove the demiclosedness principle and weak convergence theorem of the modified Mann's algorithm for $T$ in the framework of uniformly convex Banach spaces which have the Fréchet differentiable norm. Moreover, we also elicit a necessary and sufficient condition that guarantees strong convergence of the modified Mann's iterative sequence to a fixed point of $T$ in a real Banach spaces with the Fréchet differentiable norm.

We will use the notation:

1. $\rightarrow$ for weak convergence.

2. $\omega_{\mathcal{W}}\left(x_{n}\right)=\left\{x: \exists x_{n_{j}} \rightarrow x\right\}$ denotes the weak $\omega$-limit set of $\left\{x_{n}\right\}$.

\section{Preliminaries}

Let $E$ be a real Banach space. The space $E$ is called uniformly convex if for each $\varepsilon>0$, there exists a $\delta>0$ such that for $x, y \in E$ with $\|x\| \leq 1,\|y\| \leq 1,\|x-y\| \geq \varepsilon$, we have $\left\|\frac{1}{2}(x+y)\right\| \leq 1-\delta$. The modulus of convexity of $E$ is defined by

$$
\delta_{E}(\varepsilon)=\inf \left\{1-\left\|\frac{1}{2}(x+y)\right\|:\|x\| \leq 1,\|y\| \leq 1,\|x-y\| \geq \varepsilon,\right\} \forall x, y \in E
$$

for all $\varepsilon \in[0,2] . E$ is uniformly convex if $\delta_{E}(0)=0$ and $\delta_{E}(\varepsilon)>0$ for all $\varepsilon \in(0,2]$. The modulus of smoothness of $E$ is the function $\rho_{E}:[0, \infty) \in[0, \infty)$ defined by

$$
\rho_{E}(\tau)=\sup \left\{\frac{1}{2}(\|x+y\|+\|x-y\|)-1:\|x\| \leq 1,\|y\| \leq \tau\right\}, \forall x, y \in E .
$$

$E$ is uniformly smooth if and only if $\lim _{\tau \rightarrow 0} \frac{\rho_{E}(\tau)}{\tau}=0$.

$E$ is said to have a Fréchet differentiable norm if for all $x \in U=\{x \in E:\|x\|=1\}$

$$
\lim _{t \rightarrow 0} \frac{\|x+t y\|-\|x\|}{t}
$$

exists and is attained uniformly in $y \in U$. In this case, there exists an increasing function $b:[0, \infty) \rightarrow[0, \infty)$ with $\lim _{t \rightarrow 0}[b(t) / t]=0$ such that for all $x, h \in E$

$$
\frac{1}{2}\|x\|^{2}+\langle h, j(x)\rangle \leq \frac{1}{2}\|x+h\|^{2} \leq \frac{1}{2}\|x\|^{2}+\langle h, j(x)\rangle+b(\|h\|) .
$$

It is well known (see, for example, [[12], p. 107]) that uniformly smooth Banach space has a Fréchet differentiable norm.

Lemma 2.1 [2, p. 80] Let $\left\{a_{n}\right\}_{n=1}^{\infty},\left\{b_{n}\right\}_{n=1}^{\infty},\left\{\delta_{n}\right\}_{n=1}^{\infty}$ be nonnegative sequences of real numbers satisfying the following inequality

$$
a_{n+1} \leq\left(1+\delta_{n}\right) a_{n}+b_{n}, \forall n \geq 1 .
$$

If $\sum_{n=1}^{\infty} \delta_{n}<\infty$ and $\sum_{n=1}^{\infty} b_{n}<\infty$, then $\lim _{n \rightarrow \infty} a_{n}$ exists. If in addition $\left\{a_{n}\right\}_{n=1}^{\infty}$ has a subsequence which converges strongly to zero, then $\lim _{n \rightarrow \infty} a_{n}=0$.

Lemma 2.2 [2, p. 78] Let $E$ be a real Banach space, $K$ a nonempty subset of $E$, and $T$ : $K \rightarrow K$ an asymptotically $\kappa$-strictly pseudocontractive mapping. Then, $T$ is uniformly $L$ Lipschitzian.

Lemma 2.3 [[13], p. 29] Let $K$ be a nonempty, closed, convex, and bounded subset of a uniformly convex Banach space $E$, and let $T: K \rightarrow E$ be a nonexpansive mappings. Let $\left\{x_{n}\right\}$ be a sequence in $K$ such that $\left\{x_{n}\right\}$ converges weakly to some point $x \in K$. 
Then, there exists an increasing continuous function $h:[0, \infty) \rightarrow[0, \infty)$ with $h(0)=0$ depending on the diameter of $K$ such that

$$
h(\|x-T x\|) \leq \liminf _{n \rightarrow \infty}\left\|x_{n}-T x_{n}\right\| .
$$

Lemma 2.4 [[14], p. 9] Let $E$ be a real Banach space with the Fréchet differentiable norm.

For $x \in E$, let $\beta^{*}(t)$ be defined for $0<t<\infty$ by

$$
\beta^{*}(t)=\sup _{y \in U}\left|\frac{\|x+t y\|^{2}-\|x\|^{2}}{t}-2\langle y, j(x)\rangle\right| .
$$

Then, $\lim _{t \rightarrow 0}+\beta^{*}(t)=0$ and

$$
\|x+h\|^{2} \leq\|x\|^{2}+2\langle h, j(x)\rangle+\|h\| \beta^{*}(\|h\|), \forall h \in E \backslash\{0\} .
$$

Remark 2.5 In a real Hilbert space, we can see that $\beta^{*}(t)=t$ for $t>0$. In our more general setting, throughout this article we will still assume that

$$
\beta^{*}(t) \leq 2 t,
$$

where $\beta^{*}$ is a function appearing in (6).

Then, we prove the demiclosedness principle of $T$ in the uniformly convex Banach space which has the Fréchet differentiable norm.

Lemma 2.6 Let $E$ be a real uniformly convex Banach space which has the Fréchet differentiable norm. Let $K$ be a nonempty, closed, and convex subset of $E$ and $T: K \rightarrow$ $K$ an asymptotically $\kappa$-strictly pseudocontractive mapping with $F(T) \neq \varnothing$. Then, $(I-T)$ is demiclosed at 0 .

Proof. Let $\left\{x_{n}\right\}$ be a sequence in $K$ which converges weakly to $p \in K$ and $\left\{x_{n}-T x_{n}\right\}$ converges strongly to 0 . We prove that $(I-T)(p)=0$. Let $x^{*} \in F(T)$. Then, there exists a constant $r>0$ such that $\left\|x_{n}-x^{*}\right\| \leq r, \forall n \geq 1$. Let $\bar{B}_{r}=\left\{x \in E:\left\|x-x^{*}\right\| \leq r\right\}$, and let $C=K \cap \bar{B}_{r}$. Then, $C$ is nonempty, closed, convex, and bounded, and $\left\{x_{n}\right\} \subseteq C$. Choose any $\alpha \in(0, \kappa)$ and let $T_{\alpha, n}: K \rightarrow K$ be defined for all $x \in K$ by

$$
T_{\alpha, n} x=(1-\alpha) x+\alpha T^{n} x, n \geq 1,
$$

Then for all $x, y \in K$,

$$
\begin{aligned}
\left\|T_{\alpha, n} x-T_{\alpha, n} y\right\|^{2} & =\left\|(x-y)-\alpha\left[\left(I-T^{n}\right) x-\left(I-T^{n}\right) y\right]\right\|^{2} \\
& \leq\|x-y\|^{2}-2 \alpha\left\langle\left(I-T^{n}\right) x-\left(I-T^{n}\right) y, j(x-y)\right\rangle \\
& +\alpha\left\|x-y-\left(T^{n} x-T^{n} y\right)\right\| \beta^{*}\left[\alpha\left\|x-y-\left(T^{n} x-T^{n} y\right)\right\|\right] \\
& \|x-y\|^{2}-2 \alpha\left[\kappa\left\|x-y-\left(T^{n} x-T^{n} y\right)\right\|^{2}-\left(\kappa_{n}-1\right)\|x-y\|^{2}\right] \\
& +2 \alpha^{2}\left\|x-y-\left(T^{n} x-T^{n} y\right)\right\|^{2} \\
& =\left[1+2 \alpha\left(\kappa_{n}-1\right)\right]\|x-y\|^{2}-2 \alpha(\kappa-\alpha)\left\|x-\gamma-\left(T^{n} x-T^{n} y\right)\right\|^{2} \\
& \leq \tau_{n}^{2}\|x-y\|^{2},
\end{aligned}
$$

where $\tau_{n}=\left[1+2 \alpha\left(\kappa_{n}-1\right)\right]^{\frac{1}{2}}$. (In fact, in (7) the domain of $\beta^{*}(\cdot)$ requires $\| x-y-$ $\left(T^{n} x-T^{n} y\right) \| \neq 0$. But when $\left\|x-y-\left(T^{n} x-T^{n} y\right)\right\|=0$, we have $\left\|T_{\alpha, n} x-T_{\alpha, n} y\right\|^{2}=\|x-y\|^{2}$, which still satisfies the inequality $\left\|T_{\alpha, n} x-T_{\alpha, n} y\right\|^{2} \leq \tau_{n}^{2}\|x-y\|^{2}$. So we do not specially emphasize the situation that the argument of $\beta^{*}(\cdot)$ equals 0 in this inequality and the 
following proof of Theorem 3.1.) Define $G_{\alpha, m}: K \rightarrow E$ by

$$
G_{\alpha, m} x=\frac{1}{\tau_{m}} T_{\alpha, m} x, \quad m \geq 1 .
$$

Then, $G_{\alpha, m}$ is nonexpansive and it follows from Lemma 2.3 that there exists an increasing continuous function $h:[0, \infty) \rightarrow[0, \infty)$ with $h(0)=0$ depending on the diameter of $K$ such that

$$
h\left(\left\|p-G_{\alpha, m} p\right\|\right) \leq \liminf _{n \rightarrow \infty}\left\|x_{n}-G_{\alpha, m} x_{n}\right\| .
$$

Observe that

$$
\begin{aligned}
\left\|x_{n}-G_{\alpha, m} x_{n}\right\| & =\left\|x_{n}-\frac{1}{\tau_{m}} T_{\alpha, m} x_{n}\right\| \\
& \leq\left\|x_{n}-T_{\alpha, m} x_{n}\right\|+\left(1-\frac{1}{\tau_{m}}\right)\left(\tau_{m}\left\|x_{n}-x^{*}\right\|+\left\|x^{*}\right\|\right) \\
& \leq\left\|x_{n}-T_{\alpha, m} x_{n}\right\|+\left(1-\frac{1}{\tau_{m}}\right)\left(\tau_{m} r+\left\|x^{*}\right\|\right),
\end{aligned}
$$

and as $n \rightarrow \infty$

$$
\left\|x_{n}-T_{\alpha, m} x_{n}\right\|=\alpha\left\|x_{n}-T^{m} x_{n}\right\| \leq \sum_{j=1}^{m}\left\|T^{j-1} x_{n}-T^{j} x_{n}\right\| \leq[1+L(m-1)]\left\|x_{n}-T x_{n}\right\| \rightarrow 0 .
$$

Thus, it follows from (9) and (10) that

$$
\limsup _{n \rightarrow \infty}\left\|x_{n}-G_{\alpha, m} x_{n}\right\| \leq\left(1-\frac{1}{\tau_{m}}\right)\left(\tau_{m} r+\left\|x^{*}\right\|\right),
$$

so that (8) implies that

$$
h\left(\left\|p-G_{\alpha, m} p\right\|\right) \leq\left(1-\frac{1}{\tau_{m}}\right)\left(\tau_{m} r+\left\|x^{*}\right\|\right) .
$$

Observe that

$$
\begin{aligned}
\left\|p-G_{\alpha, m} p\right\| & \geq\left\|p-T_{\alpha, m} p\right\|-\left(1-\frac{1}{\tau_{m}}\right)\left\|T_{\alpha, m} p\right\| \\
& \geq\left\|p-T_{\alpha, m} p\right\|-\left(1-\frac{1}{\tau_{m}}\right)\left(\tau_{m} r+\left\|x^{*}\right\|\right),
\end{aligned}
$$

so that

$$
\begin{aligned}
\left\|p-T_{\alpha, m} p\right\| & \leq\left\|p-G_{\alpha, m} p\right\|+\left(1-\frac{1}{\tau_{m}}\right)\left(\tau_{m} r+\left\|x^{*}\right\|\right) \\
& \leq h^{-1}\left[\left(1-\frac{1}{\tau_{m}}\right)\left(\tau_{m} r+\left\|x^{*}\right\|\right)\right]+\left(1-\frac{1}{\tau_{m}}\right)\left(\tau_{m} r+\left\|x^{*}\right\|\right) \rightarrow 0, \text { as } m \rightarrow \infty .
\end{aligned}
$$

Since $T$ is continuous, we have $(I-T)(p)=0$, completing the proof of Lemma 2.6.

Lemma 2.7 Let $E$ be a real uniformly convex Banach space which has the Fréchet differentiable norm, and let $K$ be a nonempty, closed, and convex subset of $E$. Let $T$ : $K \rightarrow K$ be an asymptotically $\kappa$-strictly pseudocontractive mapping with $F(T) \neq \varnothing$. Let $\left\{x_{n}\right\}_{n=1}^{\infty}$ be the sequence satisfying the following conditions: 


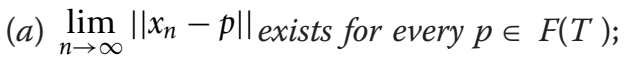

(b) $\lim _{n \rightarrow \infty}\left\|x_{n}-T x_{n}\right\|=0$;

(c) $\lim _{n \rightarrow \infty}\left\|t x_{n}+(1-t) p_{1}-p_{2}\right\|$ exists for all $t \in[0,1]$ and for all $p_{1}, p_{2} \in F(T)$.

Then, the sequence $\left\{x_{n}\right\}$ converges weakly to a fixed point of $T$.

Proof. Since $\lim _{n \rightarrow \infty}\left\|x_{n}-p\right\|$ exists, then $\left\{x_{n}\right\}$ is bounded. By (b) and Lemma 2.6, we have $\omega_{\mathcal{W}}\left(x_{n}\right) \subset F(T)$. Assume that $p_{1}, p_{2} \in \omega_{\mathcal{W}}\left(x_{n}\right)$ and that $\left\{x_{n_{i}}\right\}$ and $\left\{x_{m_{j}}\right\}$ are subsequences of $\left\{x_{n}\right\}$ such that $x_{n_{i}} \rightarrow p_{1}$ and $x_{m_{j}} \rightarrow p_{2}$, respectively. Since $E$ has the Fréchet differentiable norm, by setting $x=p_{1}-p_{2}, h=t\left(x_{n}-p_{1}\right)$ in (5) we obtain

$$
\begin{aligned}
\frac{1}{2}\left\|p_{1}-p_{2}\right\|^{2}+t\left\langle x_{n}-p_{1}, j\left(p_{1}-p_{2}\right)\right\rangle & \leq \frac{1}{2}\left\|t x_{n}+(1-t) p_{1}-p_{2}\right\|^{2} \\
& \leq \frac{1}{2}\left\|p_{1}-p_{2}\right\|^{2}+t\left\langle x_{n}-p_{1}, j\left(p_{1}-p_{2}\right)\right\rangle+b\left(t\left\|x_{n}-p_{1}\right\|\right),
\end{aligned}
$$

where $b$ is an increasing function. Since ||$x_{n}-p_{1} \| \leq M, \forall n \geq 1$, for some $M>0$, then

$$
\begin{aligned}
\frac{1}{2}\left\|p_{1}-p_{2}\right\|^{2}+t\left\langle x_{n}-p_{1}, j\left(p_{1}-p_{2}\right)\right\rangle & \leq \frac{1}{2}\left\|t x_{n}+(1-t) p_{1}-p_{2}\right\|^{2} \\
& \leq \frac{1}{2}\left\|p_{1}-p_{2}\right\|^{2}+t\left\langle x_{n}-p_{1}, j\left(p_{1}-p_{2}\right)\right\rangle+b(t M) .
\end{aligned}
$$

Therefore,

$$
\begin{aligned}
\frac{1}{2}\left\|p_{1}-p_{2}\right\|^{2}+t \limsup _{n \rightarrow \infty}\left\langle x_{n}-p_{1}, j\left(p_{1}-p_{2}\right)\right\rangle & \leq \frac{1}{2} \lim _{n \rightarrow \infty}\left\|t x_{n}+(1-t) p_{1}-p_{2}\right\|^{2} \\
& \leq \frac{1}{2}\left\|p_{1}-p_{2}\right\|^{2}+t \liminf _{n \rightarrow \infty}\left\langle x_{n}-p_{1}, j\left(p_{1}-p_{2}\right)\right\rangle+b(t M) .
\end{aligned}
$$

Hence, $\quad \lim \sup _{n \rightarrow \infty}\left\langle x_{n}-p_{1}, j\left(p_{1}-p_{2}\right)\right\rangle \leq \lim \inf _{n \rightarrow \infty}\left\langle x_{n}-p_{1}, j\left(p_{1}-p_{2}\right)\right\rangle+\frac{b(t M)}{t}$. Since $\lim _{t \rightarrow 0^{+}} \frac{b(t M)}{t}=0$, then $\lim _{n \rightarrow \infty}\left\langle x_{n}-p_{1}, j\left(p_{1}-p_{2}\right)\right\rangle$ exists. Since $\lim _{n \rightarrow \infty}\left\langle x_{n}-p_{1}, j\left(p_{1}-p_{2}\right)\right\rangle$ $=\left\langle p-p_{1}, j\left(p_{1}-p_{2}\right)\right\rangle$, for all $p \in \omega_{\mathcal{W}}\left(x_{n}\right)$. Set $p=p_{2}$. We have $\left\langle p_{2}-p_{1}, j\left(p_{1}-p_{2}\right)\right\rangle=0$, that is, $p_{2}=p_{1}$. Hence, $\omega_{\mathcal{W}}\left(x_{n}\right)$ is singleton, so that $\left\{x_{n}\right\}$ converges weakly to a fixed point of $T$.

\section{Main results}

Theorem 3.1 Let $E$ be a real uniformly convex Banach space which has the Fréchet differentiable norm, and let $K$ be a nonempty, closed, and convex subset of $E$. Let $T$ : $K \rightarrow K$ be an asymptotically $\kappa$-strictly pseudocontractive mapping for some $0 \leq \kappa<1$ with sequence $\left\{\kappa_{n}\right\}_{n=1}^{\infty} \subset[1, \infty)$, such that $\sum_{n=1}^{\infty}\left(\kappa_{n}-1\right)<\infty$, and let $F(T) \neq \varnothing$. Assume that the control sequence $\left\{\alpha_{n}\right\}_{n=1}^{\infty}$ is chosen so that

(i*) $0<\alpha_{n}<\kappa, n \geq 1$

(ii*) $\sum_{n=1}^{\infty} \alpha_{n}\left(\kappa-\alpha_{n}\right)=\infty$.

Given $x_{1} \in K$, then the sequence $\left\{x_{n}\right\}_{n=1}^{\infty}$ is generated by the modified Mann's algorithm:

$$
x_{n+1}=\left(1-\alpha_{n}\right) x_{n}+\alpha_{n} T^{n} x_{n},
$$

converges weakly to a fixed point of $T$. 
Proof. Pick a $p \in F(T)$. We firstly show that $\lim _{n \rightarrow \infty}\left\|x_{n}-p\right\|$ exists. To see this, using (2) and (6), we obtain

$$
\begin{aligned}
\left\|x_{n+1}-p\right\|^{2} & =\left\|\left(x_{n}-p\right)-\alpha_{n}\left(x_{n}-T^{n} x_{n}\right)\right\|^{2} \\
& \leq\left\|x_{n}-p\right\|^{2}-2 \alpha_{n}\left\langle x_{n}-T^{n} x_{n}, j\left(x_{n}-p\right)\right\rangle+\alpha_{n}\left\|x_{n}-T^{n} x_{n}\right\| \beta^{*}\left(\alpha_{n}\left\|x_{n}-T^{n} x_{n}\right\|\right) \\
& \leq\left\|x_{n}-p\right\|^{2}-2 \alpha_{n}\left[\kappa\left\|x_{n}-T^{n} x_{n}\right\|^{2}-\left(\kappa_{n}-1\right)\left\|x_{n}-p\right\|^{2}\right]+2 \alpha_{n}^{2}\left\|x_{n}-T^{n} x_{n}\right\|^{2} \\
& =\left[1+2 \alpha_{n}\left(\kappa_{n}-1\right)\right]\left\|x_{n}-p\right\|^{2}-2 \alpha_{n}\left(\kappa-\alpha_{n}\right)\left\|x_{n}-T^{n} x_{n}\right\|^{2} .
\end{aligned}
$$

Obviously,

$$
\left\|x_{n+1}-p\right\|^{2} \leq\left[1+2 \alpha_{n}\left(\kappa_{n}-1\right)\right]\left\|x_{n}-p\right\|^{2} .
$$

Let $\delta_{n}=1+2 \alpha_{n}\left(\kappa_{n}-1\right)$. Since $\sum_{n=1}^{\infty}\left(\kappa_{n}-1\right)<\infty$, we have

$$
\sum_{n=1}^{\infty}\left(\delta_{n}-1\right) \leq 2 \sum_{n=1}^{\infty}\left(\kappa_{n}-1\right)<\infty
$$

then (14) implies $\lim _{n \rightarrow \infty}\left\|x_{n}-p\right\|$ exists by Lemma 2.1 (and hence the sequence \{ $\|$ $\left.x_{n}-p \|\right\}$ is bounded, that is, there exists a constant $M>0$ such that $\left.\left\|x_{n}-p\right\|<M\right)$.

Then, we prove $\lim _{n \rightarrow \infty}|| x_{n}-T x_{n} \|=0$. In fact, it follows from (13) that

$$
\begin{aligned}
\sum_{n=1}^{j} 2 \alpha_{n}\left(\kappa-\alpha_{n}\right)\left\|x_{n}-T^{n} x_{n}\right\|^{2} & \leq \sum_{n=1}^{j}\left(\left\|x_{n}-p\right\|^{2}-\left\|x_{n+1}-p\right\|^{2}\right)+\sum_{n=1}^{j}\left[2 \alpha_{n}\left(\kappa_{n}-1\right)\right]\left\|x_{n}-p\right\|^{2} \\
& \leq \sum_{n=1}^{j}\left(\left\|x_{n}-p\right\|^{2}-\left\|x_{n+1}-p\right\|^{2}\right)+\sum_{n=1}^{j}\left(\delta_{n}-1\right) M^{2} .
\end{aligned}
$$

Then,

$$
\sum_{n=1}^{\infty} 2 \alpha_{n}\left(\kappa-\alpha_{n}\right)\left\|x_{n}-T^{n} x_{n}\right\|^{2}<\left\|x_{1}-p\right\|^{2}+M^{2} \sum_{n=1}^{\infty}\left(\delta_{n}-1\right)<\infty .
$$

Since $\sum_{n=1}^{\infty} \alpha_{n}\left(\kappa-\alpha_{n}\right)=\infty$, then (15) implies that $\lim _{\inf _{n \rightarrow \infty}}|| x_{n}-T^{n} x_{n} \|=0$. Thus $\lim _{n \rightarrow \infty}\left\|x_{n}-T^{n} x_{n}\right\|=0$.

By Lemma 2.2 we know that $T$ is uniformly $L$-Lipschitzian, then there exists a constant $L>0$, such that

$$
\begin{aligned}
\left\|x_{n}-T x_{n}\right\| & \leq\left\|\mid x_{n}-T^{n} x_{n}\right\|+\left\|T^{n} x_{n}-T x_{n}\right\| \leq\left\|x_{n}-T^{n} x_{n}\right\|+L\left\|T^{n-1} x_{n}-x_{n}\right\| \\
& \leq\left\|x_{n}-T^{n} x_{n}\right\|+L\left\|T^{n-1} x_{n}-T^{n-1} x_{n-1}\right\|+L\left\|T^{n-1} x_{n-1}-x_{n}\right\| \\
& \leq\left\|x_{n}-T^{n} x_{n}\right\|+L^{2}\left\|x_{n}-x_{n-1}\right\|+L\left\|T^{n-1} x_{n-1}-x_{n-1}\right\|+L\left\|x_{n}-x_{n-1}\right\| \\
& \leq\left\|x_{n}-T^{n} x_{n}\right\|+L(2+L)\left\|T^{n-1} x_{n-1}-x_{n-1}\right\|
\end{aligned}
$$

Hence, $\lim _{n \rightarrow \infty}|| x_{n}-T x_{n} \|=0$.

Now we prove that for all $p_{1}, p_{2} \in F(T), \lim _{n \rightarrow \infty}|| t x_{n}+(1-t) p_{1}-p_{2} \|$ exists for all $t$ $\in[0,1]$. Let $\sigma_{n}(t)=\left\|t x_{n}+(1-t) p_{1}-p_{2}\right\|$. It is obvious that $\lim _{n \rightarrow \infty} \sigma_{n}(0)=\| p_{1}-$ $p_{2} \|$ and $\lim _{n \rightarrow \infty} \sigma_{n}(1)=\lim _{n \rightarrow \infty}|| x_{n}-p_{2} \|$ exist. So, we only need to consider the case of $t \in(0,1)$.

Define $T_{n}: K \rightarrow K$ by

$$
T_{n} x=\left(1-\alpha_{n}\right) x+\alpha_{n} T^{n} x, \quad x \in K .
$$


Then for all $x, y \in K$,

$$
\begin{aligned}
\left\|T_{n} x-T_{n} y\right\|^{2}= & \left\|(x-y)-\alpha_{n}\left[\left(I-T^{n}\right) x-\left(I-T^{n}\right) y\right]\right\|^{2} \\
\leq & \|x-y\|^{2}-2 \alpha_{n}\left\langle\left(I-T^{n}\right) x-\left(I-T^{n}\right) y, j(x-y)\right\rangle \\
& \quad+\alpha_{n}\left\|x-y-\left(T^{n} x-T^{n} y\right)\right\| \beta^{*}\left[\alpha_{n}\left\|x-y-\left(T^{n} x-T^{n} y\right)\right\|\right] \\
\leq & \|x-y\|^{2}-2 \alpha_{n}\left[\kappa\left\|x-y-\left(T^{n} x-T^{n} y\right)\right\|^{2}-\left(\kappa_{n}-1\right)\|x-y\|^{2}\right] \\
& \quad+2 \alpha_{n}^{2}\left\|x-y-\left(T^{n} x-T^{n} y\right)\right\|^{2} \\
= & {\left[1+2 \alpha_{n}\left(\kappa_{n}-1\right)\right]\|x-y\|^{2}-2 \alpha_{n}\left(\kappa-\alpha_{n}\right)\left\|x-y-\left(T^{n} x-T^{n} \gamma\right)\right\|^{2} . }
\end{aligned}
$$

By the choice of $\alpha_{n}$, we have $\left\|T_{n} x-T_{n} y\right\|^{2} \leq\left[1+2 \alpha_{n}\left(\kappa_{n}-1\right)\right]\|x-y\|^{2}$. For the convenience of the following discussing, set $\lambda_{n}=\left[1+2 \alpha_{n}\left(\kappa_{n}-1\right)\right]^{\frac{1}{2}}$, then $\left\|T_{n} x-T_{n} y\right\| \leq$ $\lambda_{n}\|x-y\|$.

Set $S_{n, m}=T_{n+m-1} T_{n+m-2} \cdots T_{n}, m \geq 1$. We have

$$
\left\|S_{n, m} x-S_{n, m} y\right\| \leq\left(\prod_{j=n}^{n+m-1} \lambda_{j}\right)\|x-y\| \text { for all } x, y \in K,
$$

and

$$
S_{n, m} x_{n}=x_{n+m}, \quad S_{n, m} p=p \text { for all } p \in F(T) .
$$

Set $b_{n, m}=\left\|S_{n, m}\left(t x_{n}+(1-t) p_{1}\right)-t S_{n, m} x_{n}-(1-t) S_{n, m} p_{1}\right\|$. If $\left\|x_{n}-p_{1}\right\|=0$ for some $n_{0}$, then $x_{n}=p_{1}$ for any $n \geq n_{0}$ so that $\lim _{n \rightarrow \infty}|| x_{n}-p_{1} \|=0$, in fact $\left\{x_{n}\right\}$ converges strongly to $p_{1} \in F(T)$. Thus, we may assume $\left\|x_{n}-p_{1}\right\|>0$ for any $n \geq 1$. Let $\delta$ denote the modulus of convexity of $E$. It is well known (see, for example, [[15], p. 108]) that

$$
\begin{aligned}
\|t x+(1-t) y\| & \leq 1-2 \min \{t,(1-t)\} \delta(\|x-y\|) \\
& \leq 1-2 t(1-t) \delta(\|x-y\|)
\end{aligned}
$$

for all $t \in[0,1]$ and for all $x, y \in E$ such that $\|x\| \leq 1,\|y\| \leq 1$. Set

$$
\begin{aligned}
& w_{n, m}=\frac{S_{n, m} p_{1}-S_{n, m}\left(t x_{n}+(1-t) p_{1}\right)}{t\left(\prod_{j=n}^{n+m-1} \lambda_{j}\right)\left\|x_{n}-p_{1}\right\|} \\
& z_{n, m}=\frac{S_{n, m}\left(t x_{n}+(1-t) p_{1}\right)-S_{n, m} x_{n}}{(1-t)\left(\prod_{j=n}^{n+m-1} \lambda_{j}\right)\left\|x_{n}-p_{1}\right\|}
\end{aligned}
$$

Then, $\left\|w_{n, m}\right\| \leq 1$ and $\left\|z_{n, m}\right\| \leq 1$ so that it follows from (16) that

$$
2 t(1-t) \delta\left(\left\|w_{n, m}-z_{n, m}\right\|\right) \leq 1-\left\|t w_{n, m}+(1-t) z_{n, m}\right\| .
$$

Observe that

$$
\left\|w_{n, m}-z_{n, m}\right\|=\frac{b_{n, m}}{t(1-t)\left(\prod_{j=n}^{n+m-1} \lambda_{j}\right)\left\|x_{n}-p_{1}\right\|}
$$

and

$$
\left\|t w_{n, m}+(1-t) z_{n, m}\right\|=\frac{\left\|S_{n, m} x_{n}-S_{n, m} p_{1}\right\|}{\left(\prod_{j=n}^{n+m-1} \lambda_{j}\right)\left\|x_{n}-p_{1}\right\|},
$$


it follows from (17) that

$$
\begin{aligned}
& 2 t(1-t)\left(\prod_{j=n}^{n+m-1} \lambda_{j}\right)\left\|x_{n}-p_{1}\right\| \delta\left(\frac{b_{n, m}}{t(1-t)\left(\prod_{j=n}^{n+m-1} \lambda_{j}\right)\left\|x_{n}-p_{1}\right\|}\right) \\
& \leq\left(\prod_{j=n}^{n+m-1} \lambda_{j}\right)\left\|x_{n}-p_{1}\right\|-\left\|S_{n, m} x_{n}-S_{n, m} p_{1}\right\|=\left(\prod_{j=n}^{n+m-1} \lambda_{j}\right)\left\|x_{n}-p_{1}\right\|-\left\|x_{n+m}-p_{1}\right\| .
\end{aligned}
$$

Since $E$ is uniformly convex, then $\frac{\delta(s)}{s}$ is nondecreasing, and since $\left(\prod_{j=n}^{n+m-1} \lambda_{j}\right)\left\|x_{n}-p_{1}\right\| \leq\left(\prod_{j=n}^{n+m-1} \lambda_{j}\right) \lambda_{n-1}|| x_{n-1}-p_{1}\left\|\leq \cdots \leq\left(\prod_{j=n}^{n+m-1} \lambda_{j}\right)\left(\prod_{j=1}^{n-1} \lambda_{j}\right)\right\| x_{1}-p_{1}\left\|=\left(\prod_{j=1}^{n+m-1} \lambda_{j}\right)\right\| x_{1}-p_{1} \|$, hence it follows from (18) that

$$
\begin{gathered}
\frac{\left(\prod_{j=1}^{n+m-1} \lambda_{j}\right)\left\|x_{1}-p_{1}\right\|}{2} \delta\left(\frac{4}{\left(\prod_{j=1}^{n+m-1} \lambda_{j}\right)\left\|x_{1}-p_{1}\right\|} b_{n, m}\right) \leq\left(\prod_{j=n}^{n+m-1} \lambda_{j}\right)\left\|x_{n}-p_{1}\right\|-\left\|x_{n+m}-p_{1}\right\| \\
\left(\text { since } t(1-t) \leq \frac{1}{4} \text { for all } t \in[0,1]\right) .
\end{gathered}
$$

Since $\lim _{n \rightarrow \infty} \prod_{j=1}^{n+m-1} \lambda_{j}=1$ and since $\delta(0)=0$ and $\lim _{n \rightarrow \infty}\left\|x_{n}-p_{1}\right\|$ exists, then the continuity of $\delta$ yields $\lim _{n \rightarrow \infty} b_{n, m}=0$ uniformly for all $m \geq 1$. Observe that

$$
\begin{aligned}
\sigma_{n+m}(t) & \leq\left\|t x_{n+m}+(1-t) p_{1}-p_{2}+\left(S_{n, m}\left(t x_{n}+(1-t) p_{1}\right)-t S_{n, m} x_{n}-(1-t) S_{n, m} p_{1}\right)\right\| \\
& +\left\|S_{n, m}\left(t x_{n}+(1-t) p_{1}\right)-t S_{n, m} x_{n}-(1-t) S_{n, m} p_{1}\right\| \\
& =\left\|S_{n, m}\left(t x_{n}+(1-t) p_{1}\right)-S_{n, m} p_{2}\right\|+b_{n, m} \\
& \leq\left(\prod_{j=n}^{n+m-1} \lambda_{j}\right)\left\|t x_{n}+(1-t) p_{1}-p_{2}\right\|+b_{n, m} \\
& =\left(\prod_{j=n}^{n+m-1} \lambda_{j}\right) \sigma_{n}(t)+b_{n, m} .
\end{aligned}
$$

Hence, $\lim \sup _{n \rightarrow \infty} \sigma_{n}(t) \leq \lim _{\inf _{n \rightarrow \infty}} \sigma_{n}(t)$, this ensures that $\lim _{n \rightarrow \infty} \sigma_{n}(t)$ exists for all $t \in(0,1)$.

Now, apply Lemma 2.7 to conclude that $\left\{x_{n}\right\}$ converges weakly to a fixed point of T.

Theorem 3.2 Let $E$ be a real Banach space with the Fréchet differentiable norm, and let $K$ be a nonempty, closed, and convex subset of $E$. Let $T: K \rightarrow K$ be an asymptotically $\kappa$-strictly pseudocontractive mapping for some $0 \leq \kappa<1$ with sequence $\left\{\kappa_{n}\right\} \subset[1$, $\infty)$ such that $\sum_{n=1}^{\infty}\left(\kappa_{n}-1\right)<\infty$, let $F(T) \neq \varnothing$. Let $\left\{\alpha_{n}\right\}$ be a real sequence satisfying the condition (11). Given $x_{1} \in K$, let $\left\{x_{n}\right\}_{n=1}^{\infty}$ be the sequence generated by the modified Mann's algorithm (12). Then, the sequence $\left\{x_{n}\right\}$ converges strongly to a fixed point of $T$ if and only if

$$
\liminf _{n \rightarrow \infty} d\left(x_{n}, F(T)\right)=0,
$$

where $d\left(x_{n}, F(T)\right)=\inf _{p \in F}(T)\left\|x_{n}-p\right\|$. 
Proof. In the real Banach space $E$ with the Fréchet differentiable norm, we still have

$$
\left\|x_{n+1}-p\right\|^{2} \leq \delta_{n}\left\|x_{n}-p\right\|^{2} .
$$

as we have already proved in Theorem 3.1. Thus, $\left[d\left(x_{n+1}-p\right)\right]^{2} \leq \delta_{n}\left[d\left(x_{n}-p\right)\right]^{2}$ and it follows from Lemma 2.1 that $\lim _{n-\infty} d\left(x_{n}, F(T)\right)$ exists.

Now if $\left\{x_{n}\right\}$ converges strongly to a fixed point $p$ of $T$, then $\lim _{n \rightarrow \infty}\left\|x_{n}-p\right\|=0$. Since

$$
0 \leq d\left(x_{n}, F(T)\right) \leq\left\|x_{n}-p\right\|,
$$

we have $\lim _{\inf _{n \rightarrow \infty}} d\left(x_{n}, F(T)\right)=0$.

Conversely, suppose $\lim _{\inf _{n \rightarrow \infty}} d\left(x_{n}, F(T)\right)=0$, then the existence of $\lim _{n \rightarrow \infty} d\left(x_{n}\right.$, $F(T))$ implies that $\lim _{n \rightarrow \infty} d\left(x_{n}, F(T)\right)=0$. Thus, for arbitrary $\varepsilon>0$ there exists a positive integer $n_{0}$ such that $d\left(x_{n}, F(T)\right)<\frac{\varepsilon}{2}$ for any $n \geq n_{0}$.

From (19), we have

$$
\left\|x_{n+1}-p\right\|^{2} \leq\left\|x_{n}-p\right\|^{2}+M^{2}\left(\delta_{n}-1\right), n \geq 1,
$$

and for some $M>0,\left\|x_{n}-p\right\|<M$. Now, an induction yields

$$
\begin{aligned}
\left\|x_{n}-p\right\|^{2} & \leq\left\|x_{n-1}-p\right\|^{2}+M^{2}\left(\delta_{n-1}-1\right) \\
& \leq\left\|x_{n-2}-p\right\|^{2}+M^{2}\left(\delta_{n-2}-1\right)+M^{2}\left(\delta_{n-1}-1\right) \\
& \leq \cdots \leq\left\|x_{l}-p\right\|^{2}+M^{2} \sum_{j=l}^{n-1}\left(\delta_{j}-1\right), \quad n-1 \geq l \geq 1,
\end{aligned}
$$

Since $\sum_{n=1}^{\infty}\left(\delta_{n}-1\right)<\infty$, then there exists a positive integer $n_{1}$ such that $\sum_{j=n}^{\infty}\left(\delta_{j}-1\right)<\left(\frac{\varepsilon}{2 M}\right)^{2}, \forall n \geq n_{1}$. Choose $N=\max \left\{n_{0}, n_{1}\right\}$, then for all $n, m \geq N+1$ and for all $p \in F(T)$ we have

$$
\begin{aligned}
\left\|x_{n}-x_{m}\right\| & \leq\left\|x_{n}-p\right\|+\left\|x_{m}-p\right\| \\
& \leq\left[\left\|x_{N}-p\right\|^{2}+M^{2} \sum_{j=N}^{n-1}\left(\delta_{j}-1\right)\right]^{\frac{1}{2}}+\left[\left\|x_{N}-p\right\|^{2}+M^{2} \sum_{j=N}^{m-1}\left(\delta_{j}-1\right)\right]^{\frac{1}{2}} \\
& \leq\left[\left\|x_{N}-p\right\|^{2}+M^{2} \sum_{j=N}^{\infty}\left(\delta_{j}-1\right)\right]^{\frac{1}{2}}+\left[\left\|x_{N}-p\right\|^{2}+M^{2} \sum_{j=N}^{\infty}\left(\delta_{j}-1\right)\right]^{\frac{1}{2}} .
\end{aligned}
$$

Taking infimum over all $p \in F(T)$, we obtain

$$
\begin{aligned}
\left\|x_{n}-x_{m}\right\| & \leq\left\{\left[d\left(x_{N}, F(T)\right)\right]^{2}+M^{2} \sum_{j=N}^{\infty}\left(\delta_{j}-1\right)\right\}^{\frac{1}{2}}+\left\{\left[d\left(x_{N}, F(T)\right)\right]^{2}+M^{2} \sum_{j=N}^{\infty}\left(\delta_{j}-1\right)\right\}^{\frac{1}{2}} \\
& <2\left[\left(\frac{\varepsilon}{2}\right)^{2}+M^{2}\left(\frac{\varepsilon}{2 M}\right)^{2}\right]^{\frac{1}{2}}<2 \varepsilon .
\end{aligned}
$$

Thus, $\left\{x_{n}\right\}_{n=0}^{\infty}$ is Cauchy. We can also prove $\lim _{n \rightarrow \infty}|| x_{n}-T x_{n} \|=0$ as we have done in Theorem 3.1. Suppose $\lim _{n \rightarrow \infty} x_{n}=u$. Then,

$$
0 \leq\|u-T u\| \leq\left\|u-x_{n}\right\|+\left\|x_{n}-T x_{n}\right\|+L\left\|x_{n}-u\right\| \rightarrow 0 \text {, as } n \rightarrow \infty .
$$

Thus, $u \in F(T)$. 


\section{Acknowledgements}

This study was supported by the Youth Teacher Foundation of North China Electric Power University.

\section{Author details}

${ }^{1}$ School of Mathematics and Physics, North China Electric Power University, Baoding, Hebei 071003, P.R. China ${ }^{2}$ School of Economics, Renmin University of China, Beijing 100872, P.R. China ${ }^{3}$ Easyway Company Limited, Beijing 100872, P.R. China

\section{Authors' contributions}

All authors read and approved the final manuscript.

\section{Competing interests}

The authors declare that they have no competing interests.

Received: 4 May 2011 Accepted: 9 December 2011 Published: 9 December 2011

\section{References}

1. Osilike, MO: Iterative approximations of fixed points of asymptotically demicontractive mappings. Indian J Pure Appl Math. 29(12), 1291-1300 (1998)

2. Osilike, MO, Aniagbosor, SC, Akuchu, BG: Fixed points of asymptotically demicontractive mappings in arbitrary Banach spaces. Pan Am Math J. 12(2), 77-88 (2002)

3. Qihou, L: Convergence theorems of the sequence of iterates for asymptotically demicontractive and hemicontractive mappings. Nonlinear Anal. 26(11), 1835-1842 (1996). doi:10.1016/0362-546X(94)00351-H

4. Browder, FE, Petryshyn, WV: Construction of fixed points of nonlinear mappings in Hilbert space. J Math Anal Appl. 20, 197-228 (1967). doi:10.1016/0022-247X(67)90085-6

5. Osilike, MO, Udomene, A, Igbokwe, DI, Akuchu, BG: Demiclosedness principle and convergence theorems for $k$-strictly asymptotically pseudo-contractive maps. J Math Anal Appl. 326, 1334-1345 (2007). doi:10.1016/j.jmaa.2005.12.052

6. Kim, TH, Xu, HK: Convergence of the modified Mann's iteration method for asymptotically strict pseudocontractions. Nonlinear Anal. 68, 2828-2836 (2008). doi:10.1016/j.na.2007.02.029

7. Schu, J: Iterative construction of fixed points of asymptotically nonexpansive mappings. J Math Anal Appl. 158, 407-413 (1991). doi:10.1016/0022-247X(91)90245-U

8. Schu, J: Weak and strong convergence of fixed points of asymptotically nonexpansive mappings. Bull Aust Math Soc 43, 153-159 (1991). doi:10.1017/50004972700028884

9. Osilike, MO, Aniagbosor, SC: Weak and strong convergence theorems for fixed points of asymptotically nonexpansive mappings. J Math Comput Model. 32, 1181-1191 (2000). doi:10.1016/S0895-7177(00)00199-0

10. Tan, KK, Xu, HK: Fixed point iteration processes for asymptotically nonexpansive mappings. Proc Am Math Soc. 122(3), 733-739 (1994). doi:10.1090/50002-9939-1994-1203993-5

11. Huang, Z: Mann and Ishikawa iterations with errors for asymptotically nonexpansive mappings. Comput Math Appl. 37, $1-7$ (1999)

12. Takahashi, W: Nonlinear Functional Analysis: Fixed Point Theory and Its Applications. Yokohama Publishers, Yokohama (2000)

13. Krüppel, M: On an inequality for nonexpansive mappings in uniformly convex Banach spaecs. Rostock Math Kolloq. 51, 25-32 (1997)

14. Cholamjiak, P, Suantai, S: Weak convergence theorems for a countable family of strict pseudocontractions in banach spaces. Fixed Point Theory Appl (2010). doi:10.1155/2010/632137

15. Bruck, RE: A simple proof of the mean ergodic theorem for nonlinear contractions in Banach spaces. Israel J Math. 32(23), 107-116 (1979). doi:10.1007/BF02764907

doi:10.1186/1687-1812-2011-100

Cite this article as: Zhang and Xie: Convergence of the modified Mann's iterative method for asymptotically $\kappa$-strictly pseudocontractive mappings. Fixed Point Theory and Applications 2011 2011:100.

\section{Submit your manuscript to a SpringerOpen ${ }^{\odot}$ journal and benefit from:}

- Convenient online submission

Rigorous peer review

- Immediate publication on acceptance

- Open access: articles freely available online

- High visibility within the field

- Retaining the copyright to your article

Submit your next manuscript at $\gg$ springeropen.com 